\title{
Greatly enhanced arsenic shoot assimilation in rice leads to elevated grain levels compared to wheat \& barley
}

Paul N. Williams, Antia Villada, Claire Deacon, Andrea Raab, Jordi Figuerola, Andrew J. Green, Jörg Feldmann, Andrew A. Meharg

Supporting Information

Pages $=22$

Figures $=6$

Tables $=13$ 


\section{Contents}

Page No.

Table $\mathbf{S 1}$ Duplicate analysis of grain samples.

Table S2 Duplicate analysis of shoot samples.

Table S3 Duplicate analysis of soil samples.

Table S4 Composition and concentration of multielement standard.

Table S5 Summary of quality control.

Table S6 Summary of As levels in wheat pot experiment.

Fig S1 Biplot and regressions of grain and shoot As level against Ti level.

Table $\mathbf{S 7} \quad$ Summary of translocation factors for rice

Table $\mathbf{S 8} \quad$ Summary of translocation factors for wheat \& barley

Fig S2 Plant to soil As transfer factors for shoot and grain for rice,

Table S9 Regression of log shoot As level against log grain As: Comparision of field and pot experiment data.

Fig S3 Median grain/shoot transfer factor for rice, wheat and barley.

Table S10 Analysis of variance of As grain level between rice, wheat and barley, with variable shoot As levels.

Table S11 Summary of regressions for Soil-Shoot-Grain As levels for rice, wheat and barley.

Fig S4 Predicting rice grain As level.

Fig S5 Predicting wheat grain As level.

Table $\mathbf{S 1 2}$ Physico-chemical properties of soil

Table S13 Kinetic parameters for arsenate and arsenite in rice and wheat

Fig S6 Sample Map

References 


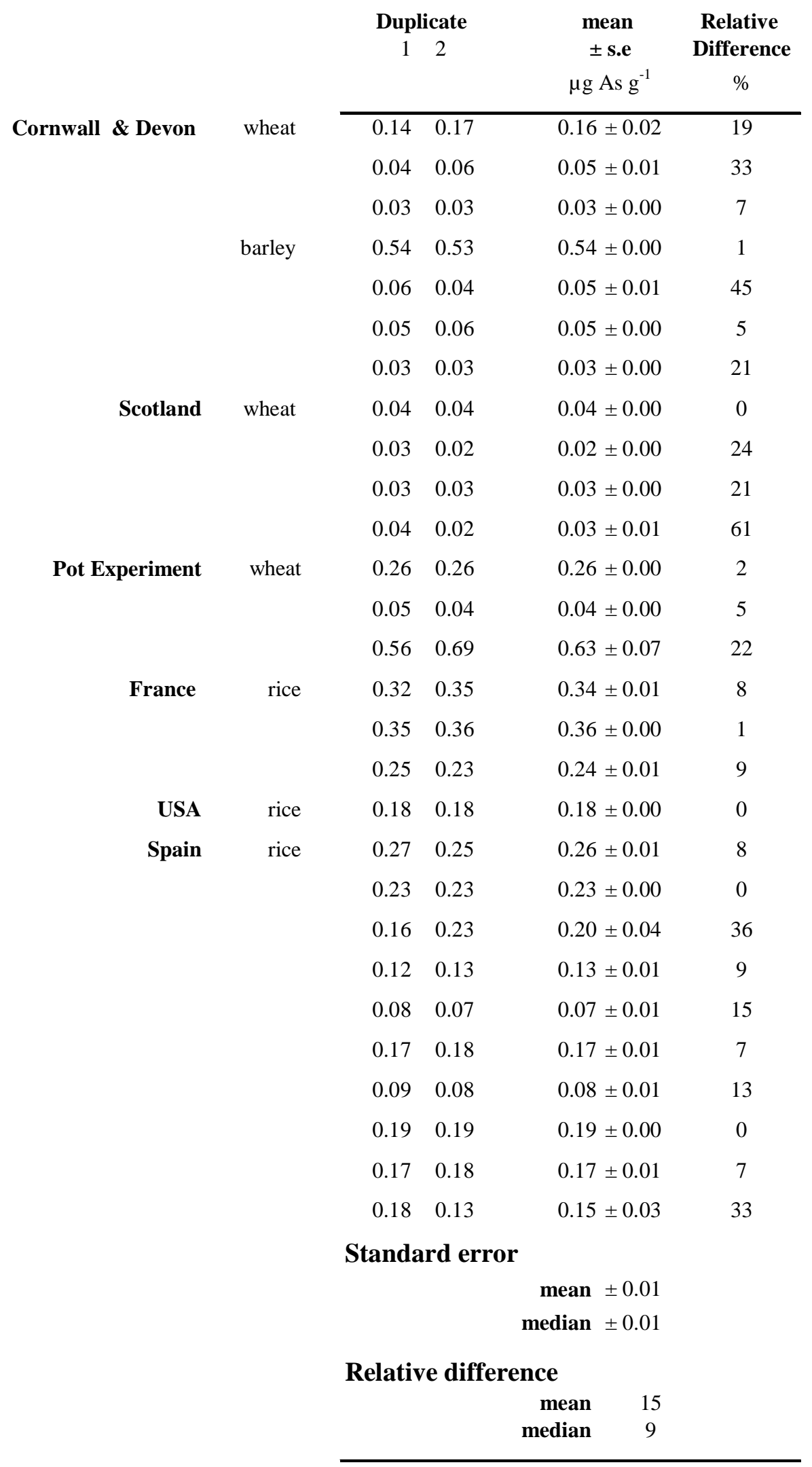

Table S1. Duplicate analysis of grain samples. Relative Difference $=($ Difference between duplicates/ duplicate mean)*100. 


\begin{tabular}{|c|c|c|c|c|c|}
\hline & & Dup & cate & mean & Relative \\
\hline & & 1 & 2 & \pm s.e & Difference \\
\hline & & & & $\mu \mathrm{g} \mathrm{As} \mathrm{g}^{-1}$ & $\%$ \\
\hline Cornwall \& Devon & wheat & 9.8 & 10.5 & $10.1 \pm 0.34$ & 7 \\
\hline & & 64.6 & 64.6 & $64.6 \pm 0.02$ & 0 \\
\hline & & 16.4 & 17.3 & $16.9 \pm 0.44$ & 5 \\
\hline & & 15.0 & 15.4 & $15.2 \pm 0.23$ & 3 \\
\hline & barley & 227.8 & 226.2 & $227.0 \pm 0.78$ & 1 \\
\hline & & 40.7 & 37.9 & $39.3 \pm 1.43$ & 7 \\
\hline & & 80.6 & 77.2 & $78.9 \pm 1.72$ & 4 \\
\hline Scotland & wheat & 7.5 & 7.7 & $7.6 \pm 0.10$ & 3 \\
\hline & & 5.7 & 6.5 & $6.1 \pm 0.40$ & 13 \\
\hline & & 3.2 & 4.0 & $3.6 \pm 0.42$ & 23 \\
\hline & & 3.2 & 2.9 & $3.0 \pm 0.18$ & 12 \\
\hline France & rice & 9.2 & 9.8 & $9.5 \pm 0.26$ & 6 \\
\hline & & 9.5 & 8.2 & $8.8 \pm 0.67$ & 15 \\
\hline & & 7.7 & 7.6 & $7.6 \pm 0.06$ & 2 \\
\hline & & 10.9 & 11.4 & $11.2 \pm 0.23$ & 4 \\
\hline USA & rice & 4.8 & 3.4 & $4.1 \pm 0.71$ & 35 \\
\hline Spain & rice & 12.2 & 11.5 & $11.9 \pm 0.37$ & 6 \\
\hline & & 5.4 & 5.6 & $5.5 \pm 0.10$ & 4 \\
\hline & & 5.6 & 5.9 & $5.8 \pm 0.15$ & 5 \\
\hline & & 5.6 & 5.5 & $5.6 \pm 0.02$ & 1 \\
\hline & & 6.1 & 6.1 & $6.1 \pm 0.02$ & 1 \\
\hline & & 8.6 & 7.9 & $8.3 \pm 0.34$ & 8 \\
\hline & & 8.0 & 7.5 & $7.7 \pm 0.22$ & 6 \\
\hline & & 2.1 & 2.0 & $2.1 \pm 0.06$ & 6 \\
\hline & & 1.5 & 1.4 & $1.4 \pm 0.08$ & 11 \\
\hline & & 1.8 & 1.8 & $1.8 \pm 0.01$ & 1 \\
\hline
\end{tabular}

Standard error
mean \pm 0.36
median \pm 0.23
Relative difference
mean
median $\quad 5$

Table S2. Duplicate analysis of shoot samples. Relative Difference $=($ Difference between duplicates/ duplicate mean)*100. 


\begin{tabular}{|c|c|c|c|c|c|}
\hline & \multicolumn{2}{|c|}{ Duplicate } & \multirow{2}{*}{$\begin{array}{c}\text { mean } \\
\pm \text { S.e }\end{array}$} & \multirow{2}{*}{$\begin{array}{l}\text { Relative } \\
\text { Difference }\end{array}$} \\
\hline & & 1 & 2 & & \\
\hline & & & & $\mu \mathrm{g} \mathrm{As} \mathrm{g}^{-1}$ & \\
\hline \multirow[t]{7}{*}{ Cornwall \& Devon } & \multirow[t]{3}{*}{ wheat } & 0.55 & 0.53 & $0.54 \pm 0.01$ & 3 \\
\hline & & 0.25 & 0.25 & $0.25 \pm 0.00$ & 3 \\
\hline & & 0.22 & 0.21 & $0.21 \pm 0.01$ & 6 \\
\hline & \multirow[t]{4}{*}{ barley } & 1.55 & 1.75 & $1.65 \pm 0.10$ & 12 \\
\hline & & 0.41 & 0.42 & $0.41 \pm 0.01$ & 4 \\
\hline & & 0.21 & 0.20 & $0.21 \pm 0.01$ & 5 \\
\hline & & 0.15 & 0.08 & $0.12 \pm 0.04$ & 61 \\
\hline \multirow[t]{4}{*}{ Scotland } & \multirow[t]{4}{*}{ wheat } & 0.22 & 0.21 & $0.21 \pm 0.00$ & 4 \\
\hline & & 0.13 & 0.13 & $0.13 \pm 0.00$ & 4 \\
\hline & & 0.09 & 0.10 & $0.10 \pm 0.00$ & 6 \\
\hline & & 0.22 & 0.24 & $0.23 \pm 0.01$ & 5 \\
\hline \multirow[t]{3}{*}{ Pot Experiment } & \multirow[t]{3}{*}{ wheat } & 0.72 & 0.71 & $0.71 \pm 0.01$ & 2 \\
\hline & & 0.06 & 0.05 & $0.05 \pm 0.00$ & 15 \\
\hline & & 3.22 & 3.15 & $3.19 \pm 0.03$ & 2 \\
\hline \multirow[t]{2}{*}{ France } & \multirow[t]{2}{*}{ rice } & 4.23 & 4.86 & $4.54 \pm 0.31$ & 14 \\
\hline & & 14.80 & 13.35 & $14.07 \pm 0.73$ & 10 \\
\hline \multirow[t]{2}{*}{ USA } & \multirow[t]{2}{*}{ rice } & 1.35 & 1.34 & $1.35 \pm 0.00$ & 1 \\
\hline & & 0.72 & 0.72 & $0.72 \pm 0.00$ & 0 \\
\hline \multirow[t]{10}{*}{ Spain } & \multirow[t]{10}{*}{ rice } & 2.32 & 2.15 & $2.24 \pm 0.09$ & 8 \\
\hline & & 9.80 & 8.76 & $9.28 \pm 0.52$ & 11 \\
\hline & & 1.12 & 1.18 & $1.15 \pm 0.03$ & 5 \\
\hline & & 1.73 & 1.42 & $1.58 \pm 0.15$ & 20 \\
\hline & & 2.83 & 1.78 & $2.30 \pm 0.52$ & 45 \\
\hline & & 2.60 & 2.23 & $2.41 \pm 0.18$ & 15 \\
\hline & & 0.44 & 0.39 & $0.42 \pm 0.02$ & 12 \\
\hline & & 1.33 & 1.70 & $1.52 \pm 0.19$ & 25 \\
\hline & & 2.87 & 2.65 & $2.76 \pm 0.11$ & 8 \\
\hline & & 1.30 & 1.24 & $1.27 \pm 0.03$ & 4 \\
\hline
\end{tabular}

Standard error

mean \pm 0.11

median \pm 0.03

Relative difference

$\begin{array}{rr}\text { mean } & 11 \\ \text { median } & 6\end{array}$

Table S3. Duplicate analysis of soil samples. Relative Difference $=($ Difference between duplicates/ duplicate mean)*100. 


\begin{tabular}{c|cccccc} 
& \multicolumn{6}{c}{ concentration, $\boldsymbol{\mu g} \mathbf{L}^{-2}$} \\
Standard & $\mathbf{C o}$ & $\mathbf{S e}$ & $\mathbf{A s}$ & $\mathbf{C u}$ & $\mathbf{Z n}$ & $\mathbf{M n}$ \\
\hline $\mathbf{1}$ & 0 & 0 & 0 & 0 & 0 & 0 \\
$\mathbf{3}$ & 0.05 & 0.25 & 0.25 & 1.25 & 2.5 & 2.5 \\
$\mathbf{4}$ & 0.2 & 1 & 1 & 5 & 10 & 10 \\
$\mathbf{5}$ & 0.4 & 2 & 2 & 10 & 20 & 20 \\
& 2 & 10 & 10 & 50 & 100 & 100
\end{tabular}

Table S4. Composition and concentration of the multielement standard. 
Total arsenic Recovery $\quad \mathbf{n}$ $\mu \mathrm{g} \mathrm{g}^{-1}$

$\begin{array}{lllll}\text { Rice flour } & \text { NIST } 1568 \mathrm{a} & \mathbf{0 . 3 0} \pm 0.003 & \mathbf{1 0 4} \pm 1 & 26\end{array}$

$\begin{array}{lllll}\text { Baseline As Soil } & \text { NCS ZC73007 } & \mathbf{1 5} \pm 0.1 & \mathbf{8 4} \pm 1 & 9 \\ \text { Elevated As Soil } & \text { GBW } 07406 & \mathbf{1 8 0} \pm 3 & \mathbf{8 2} \pm 1 & 3\end{array}$

Total arsenic Recovery $\quad \mathbf{n}$ $\mu g \mathrm{~L}^{-1} \quad \%$

$\begin{array}{rcccr}\text { Grain \& shoot } & 5 \mu \mathrm{g} \mathrm{g}^{-1} \text { spike } & \mathbf{4 . 5} \pm 0.1 & \mathbf{8 9} \pm 2 & 15 \\ \text { Grain \& shoot } & 10 \mu \mathrm{g} \mathrm{g}^{-1} \text { spike } & \mathbf{9 . 3} \pm 0.3 & \mathbf{9 3} \pm 3 & 11 \\ \text { Soil } & 10 \mu \mathrm{g} \mathrm{g}^{-1} \text { spike } & \mathbf{9 . 1} \pm 0.1 & \mathbf{9 1} \pm 1 & 5 \\ \text { Soil } & 50 \mu \mathrm{gg} \mathrm{g}^{-1} \text { spike } & \mathbf{4 4} \pm 0.9 & \mathbf{8 7} \pm 2 & 7\end{array}$

L.O.D.

$\mu \mathrm{g} \mathrm{\textrm {L } ^ { - 1 }}$

Grain

\& Shoot

$0.01-0.18$

Soil

$0.01-0.17$

Table S5. Summary of quality control. Means \pm s.e. 


\begin{tabular}{|c|c|c|c|}
\hline $\begin{array}{l}\text { Spike } \\
\mu \mathrm{g} \mathrm{g}^{-1}\end{array}$ & $\begin{array}{l}\text { Wheat } \\
\text { Cultivar }\end{array}$ & $\begin{array}{l}\text { Grain } \\
\mu \mathrm{g} \mathrm{g}^{-1}\end{array}$ & $\begin{array}{l}\text { Shoot } \\
\mu \mathrm{gg}^{-1}\end{array}$ \\
\hline \multirow[t]{6}{*}{0} & Maris Dove & 0.02 & 0.06 \\
\hline & & 0.02 & 0.05 \\
\hline & & 0.03 & 0.04 \\
\hline & Scamp & 0.03 & 0.07 \\
\hline & & 0.01 & 0.06 \\
\hline & & 0.04 & 0.06 \\
\hline \multirow[t]{6}{*}{10} & Maris Dove & 0.11 & 0.48 \\
\hline & & 0.26 & 0.72 \\
\hline & & 0.32 & 1.08 \\
\hline & Scamp & 0.20 & 0.42 \\
\hline & & 0.28 & 1.66 \\
\hline & & 0.19 & 0.51 \\
\hline \multirow[t]{6}{*}{25} & Maris Dove & 0.50 & 2.22 \\
\hline & & 0.52 & 1.98 \\
\hline & & 0.48 & 1.76 \\
\hline & Scamp & 0.40 & 1.14 \\
\hline & & 0.44 & 1.64 \\
\hline & & $\sim$ & 2.00 \\
\hline \multirow[t]{6}{*}{50} & Maris Dove & 0.51 & 1.58 \\
\hline & & 0.59 & 2.70 \\
\hline & & 0.55 & 3.62 \\
\hline & Scamp & 0.75 & 3.10 \\
\hline & & 0.71 & 5.16 \\
\hline & & 0.69 & 3.22 \\
\hline
\end{tabular}

Table S6. Summary of As levels in wheat pot experiment.

The cultivars were kindly provided to us by the John Innes Centre and have featured in numerous studies and UK crop trials $(1,2)$. Scamp is from a (Club/Moulin)/Alexandria pedigree, whilst Maris Dove is from a H 8810-47*Koga 2 cross. 


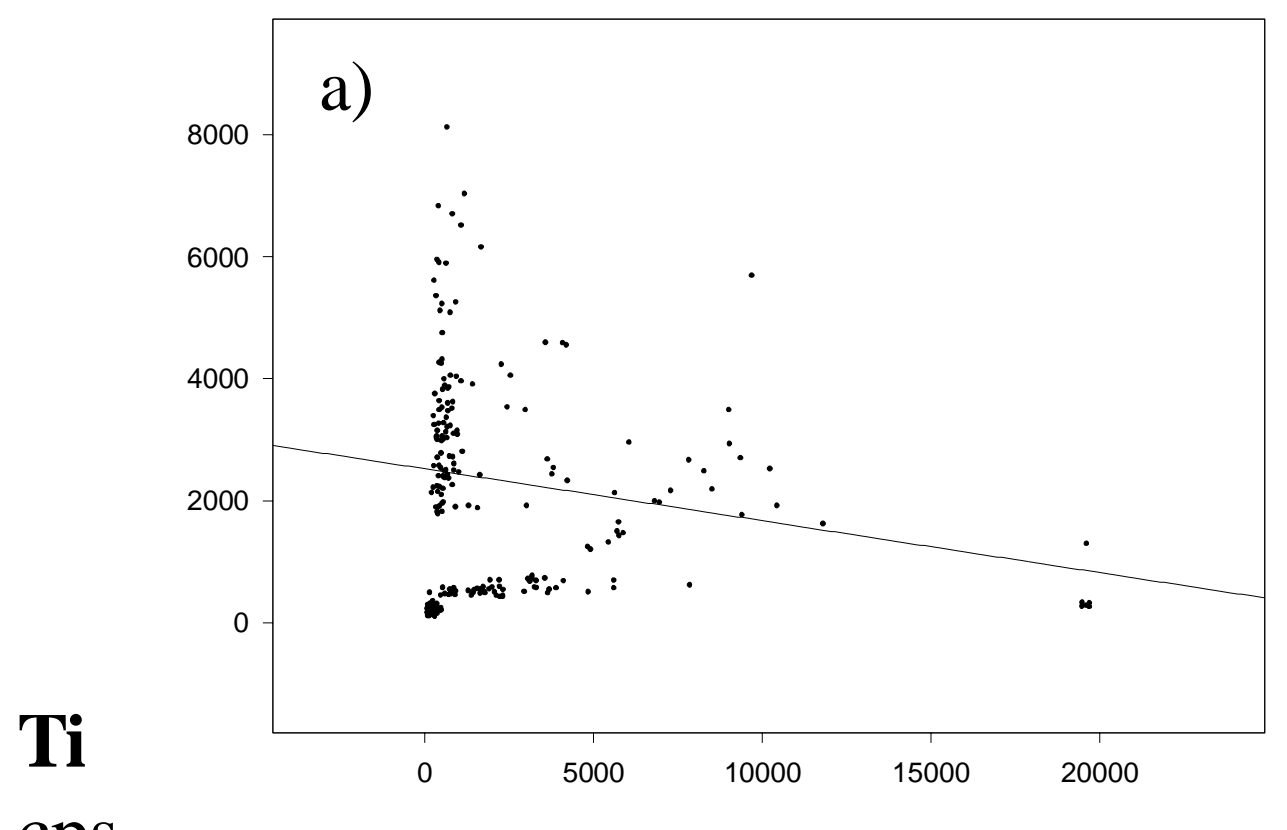

cps

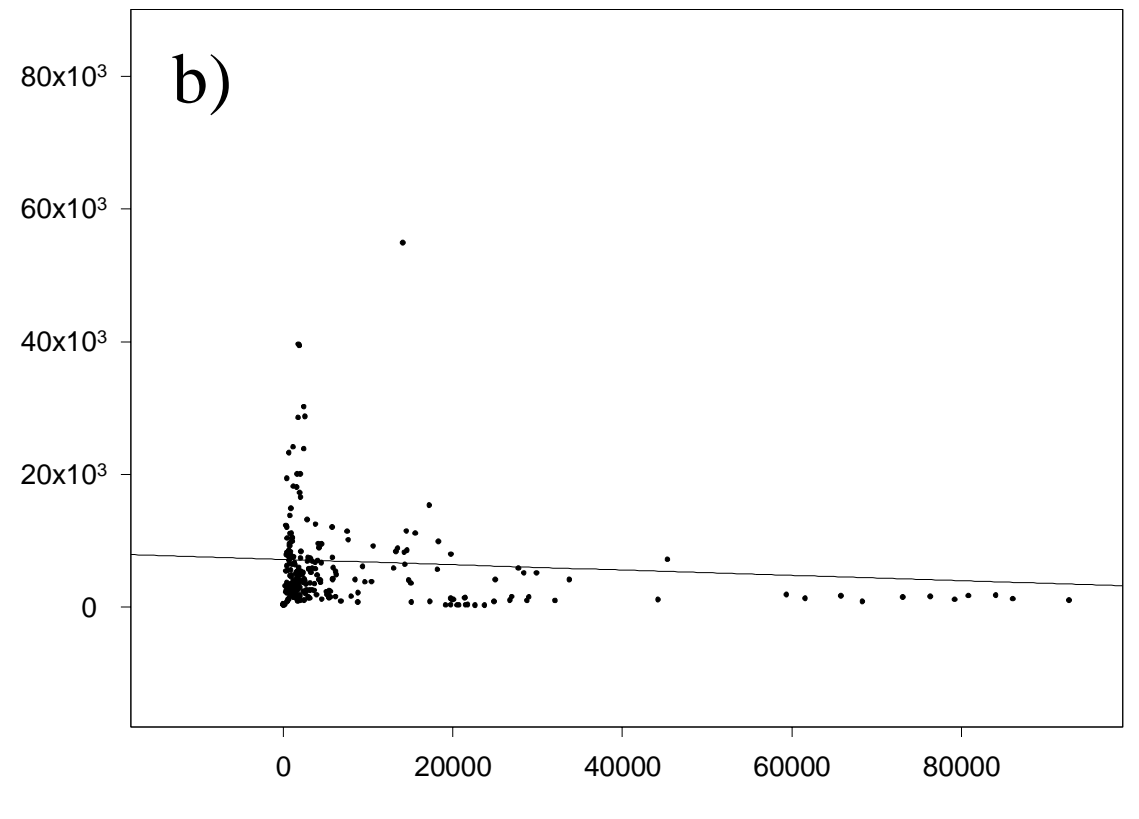

As cps

Fig S1. Biplot and regressions of relative grain and shoot As level against $\mathrm{Ti}$ level. CPS refers to counts per second

a) Grain b) Shoot. 


\begin{tabular}{|c|c|c|c|c|c|c|c|}
\hline \multirow[t]{2}{*}{ Crop } & \multirow[t]{2}{*}{ Country } & \multirow[t]{2}{*}{ Region } & & \multicolumn{4}{|c|}{ Transfer Factor } \\
\hline & & & & Min - Max & Mean & Median & $\mathrm{n}$ \\
\hline \multirow{18}{*}{$\begin{array}{l}\text { Rice } \\
(\text { Oryza) }\end{array}$} & Overall & & Grain/Soil & $0.01-0.12$ & 0.04 & 0.04 & 72 \\
\hline & & & Shoot/Soil & $0.05-3.84$ & 0.76 & 0.49 & 73 \\
\hline & & & Grain/Shoot & $0.02-0.36$ & 0.09 & 0.05 & 72 \\
\hline & France & Carmargue & Grain/Soil & $0.02-0.12$ & 0.04 & 0.04 & 22 \\
\hline & & & Shoot/Soil & $0.27-3.84$ & 1.38 & 0.95 & 23 \\
\hline & & & Grain/Shoot & $0.02-0.09$ & 0.04 & 0.03 & 22 \\
\hline & Spain & Doñana & Grain/Soil & $0.01-0.05$ & 0.02 & 0.02 & 25 \\
\hline & & & Shoot/Soil & $0.08-1.77$ & 0.47 & 0.36 & 25 \\
\hline & & & Grain/Shoot & $0.02-0.15$ & 0.06 & 0.05 & 25 \\
\hline & & Cadiz & Grain/Soil & $0.03-0.12$ & 0.07 & 0.07 & 10 \\
\hline & & & Shoot/Soil & $0.20-1.42$ & 0.76 & 0.69 & 10 \\
\hline & & & Grain/Shoot & $0.04-0.23$ & 0.12 & 0.13 & 10 \\
\hline & $U S A$ & California & Grain/Soil & $0.03-0.06$ & 0.04 & 0.04 & 9 \\
\hline & & & Shoot/Soil & $0.16-0.45$ & 0.27 & 0.26 & 9 \\
\hline & & & Grain/Shoot & $0.13-0.33$ & 0.18 & 0.16 & 9 \\
\hline & & Arkansas & Grain/Soil & $0.01-0.06$ & 0.04 & 0.03 & 6 \\
\hline & & & Shoot/Soil & $0.05-0.79$ & 0.28 & 0.22 & 6 \\
\hline & & & Grain/Shoot & $0.02-0.36$ & 0.21 & 0.23 & 6 \\
\hline
\end{tabular}

Table S7. Summary of translocation factors for rice. 


\begin{tabular}{|c|c|c|c|c|c|c|c|}
\hline \multirow[t]{2}{*}{ Crop } & \multirow[t]{2}{*}{ Country } & \multirow[t]{2}{*}{ Region } & & \multicolumn{4}{|c|}{ Transfer Factor } \\
\hline & & & & Min - Max & Mean & Median & $\mathrm{n}$ \\
\hline \multirow{9}{*}{$\begin{array}{l}\text { Wheat } \\
\text { (Triticum) }\end{array}$} & \multirow[t]{3}{*}{ Overall } & & Grain/Soil & $0.000-0.024$ & 0.004 & 0.003 & 66 \\
\hline & & & Shoot/Soil & $0.002-0.142$ & 0.018 & 0.013 & 64 \\
\hline & & & Grain/Shoot & $0.017-4.335$ & 0.30 & 0.19 & 64 \\
\hline & \multirow{3}{*}{ Scotland } & \multirow{3}{*}{ East Coast } & Grain/Soil & $0.001-0.024$ & 0.005 & 0.004 & 29 \\
\hline & & & Shoot/Soil & $0.007-0.142$ & 0.028 & 0.021 & 29 \\
\hline & & & Grain/Shoot & $0.02-1.03$ & 0.24 & 0.19 & 29 \\
\hline & \multirow[t]{3}{*}{ England } & \multirow{3}{*}{$\begin{array}{l}\text { Cornwall } \\
\text { \& Devon }\end{array}$} & Grain/Soil & $0.000-0.015$ & 0.003 & 0.002 & 37 \\
\hline & & & Shoot/Soil & $0.002-0.023$ & 0.010 & 0.009 & 35 \\
\hline & & & Grain/Shoot & $0.08-4.33$ & 0.34 & 0.20 & 35 \\
\hline \multirow{9}{*}{$\begin{array}{l}\text { Barley } \\
\text { (Hordeum) }\end{array}$} & \multirow[t]{3}{*}{ Overall } & & Grain/Soil & $0.000-0.019$ & 0.003 & 0.002 & 35 \\
\hline & & & Shoot/Soil & $0.002-0.095$ & 0.013 & 0.010 & 35 \\
\hline & & & Grain/Shoot & $0.012-1.593$ & 0.28 & 0.20 & 35 \\
\hline & \multirow[t]{3}{*}{ Scotland } & \multirow[t]{3}{*}{ East Coast } & Grain/Soil & $0.003-0.006$ & 0.005 & 0.006 & 6 \\
\hline & & & Shoot/Soil & $0.010-0.030$ & 0.019 & 0.017 & 6 \\
\hline & & & Grain/Shoot & $0.20-0.43$ & 0.31 & 0.33 & 6 \\
\hline & \multirow[t]{3}{*}{ England } & \multirow{3}{*}{$\begin{array}{l}\text { Cornwall } \\
\& \text { Devon }\end{array}$} & Grain/Soil & $0.000-0.019$ & 0.002 & 0.002 & 29 \\
\hline & & & Shoot/Soil & $0.002-0.095$ & 0.012 & 0.009 & 29 \\
\hline & & & Grain/Shoot & $0.01-1.59$ & 0.27 & 0.18 & 29 \\
\hline
\end{tabular}

Table S8. Summary of translocation factors for wheat \& barley. 


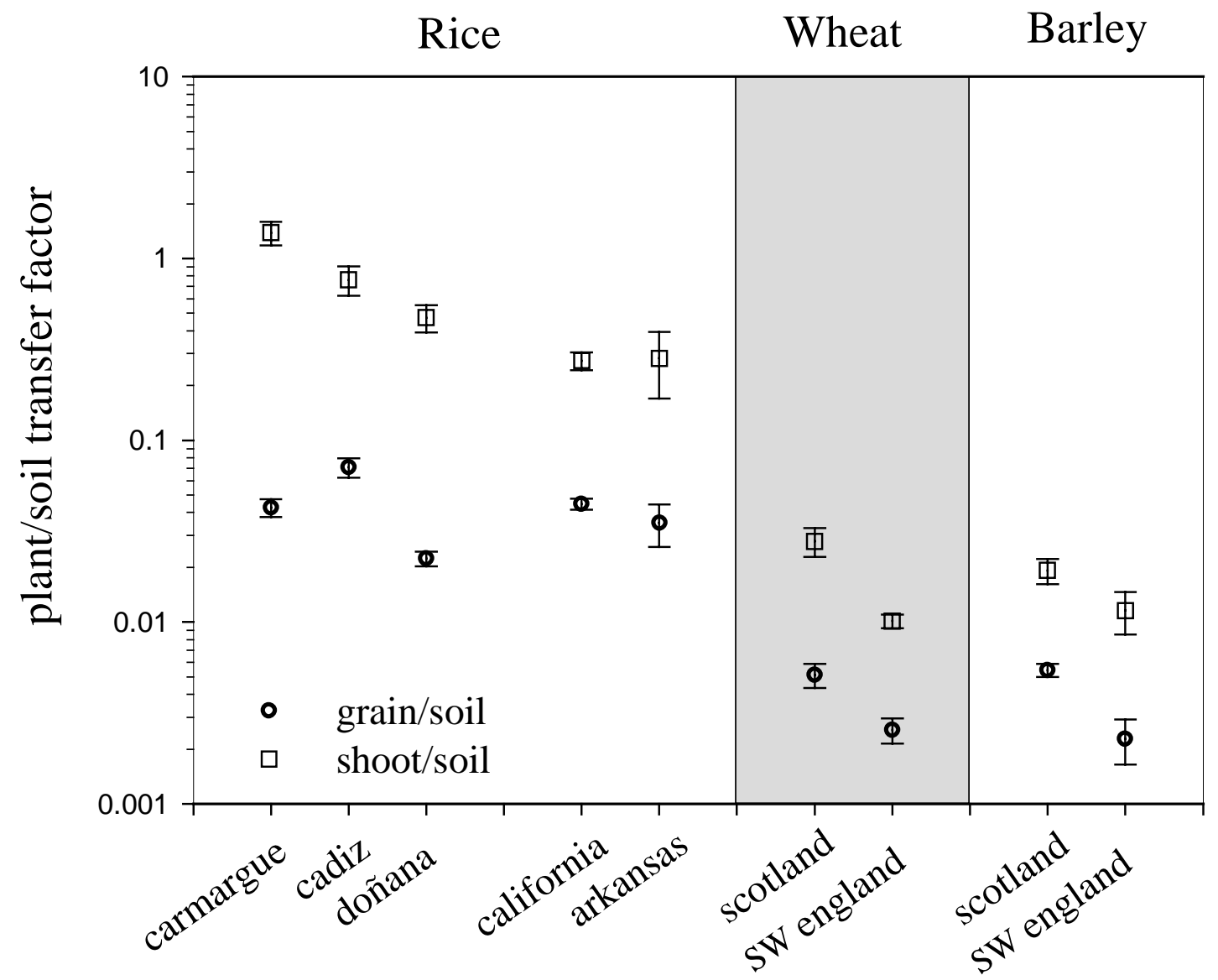

Fig S2. Plant to soil As transfer factors for shoot and grain for rice, wheat and barley. Average \pm s.e. 


\begin{tabular}{|c|c|c|c|c|c|c|}
\hline & & $\begin{array}{c}\text { R sqrt } \\
\% \\
\end{array}$ & y0 & $\mathbf{a}$ & $\mathbf{p}$ & $\mathbf{n}$ \\
\hline \multirow[t]{2}{*}{ Rice } & Field & 57.5 & -0.92 & 0.38 & $<0.001$ & 72 \\
\hline & Pot & 68.3 & -1.04 & 0.36 & $<0.001$ & 24 \\
\hline \multirow[t]{2}{*}{ Wheat } & Field & 36.7 & -1.00 & 0.59 & $<0.001$ & 64 \\
\hline & Pot & 94.2 & -0.57 & 0.83 & $<0.001$ & 23 \\
\hline
\end{tabular}

Table S9. Regression of log shoot As level against log grain As: Comparison of field and pot experiment data. 


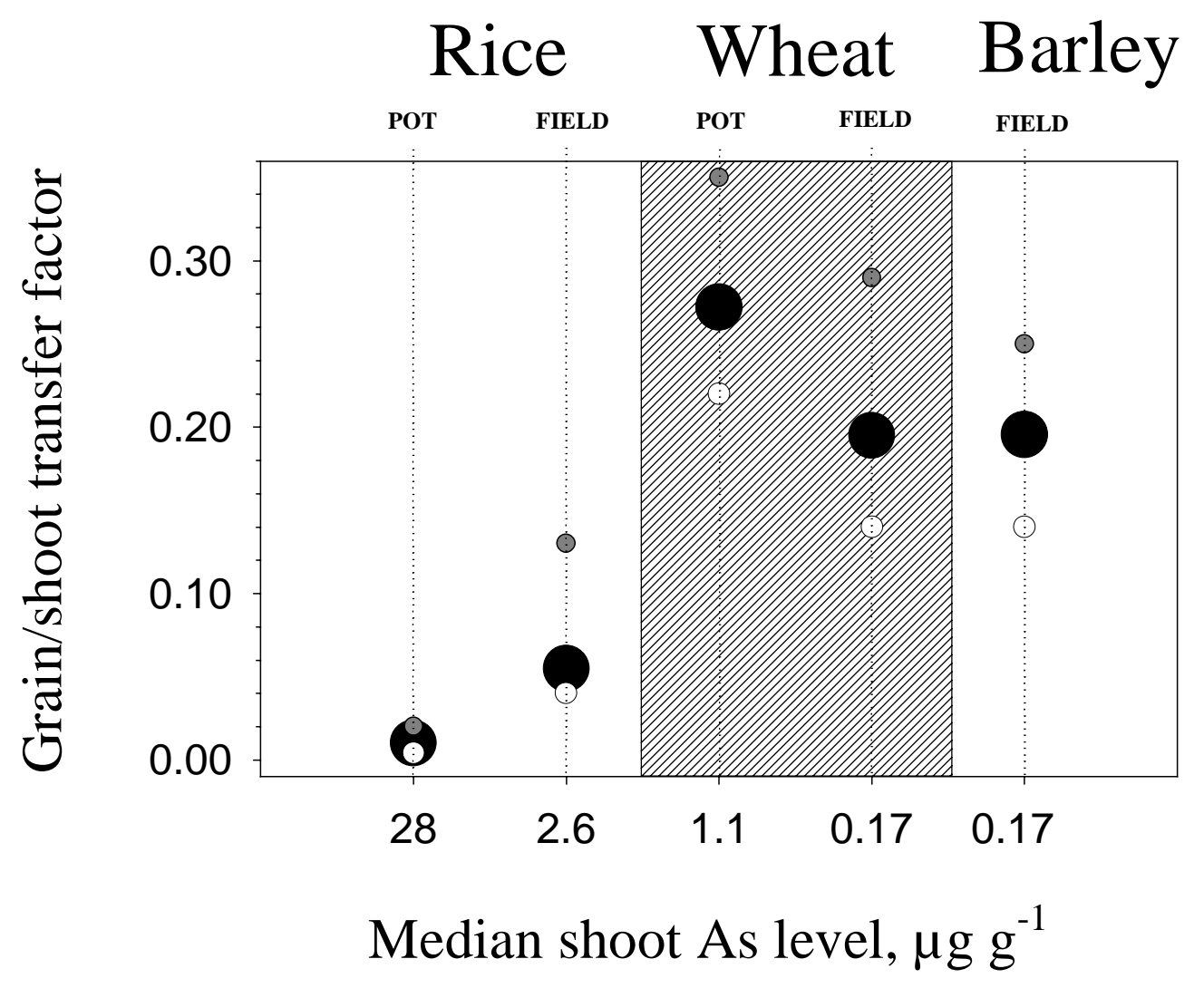

Fig S3. Median grain/shoot transfer factor for rice, wheat and barley. Median (black) $25^{\text {th }}$ (white) and $75^{\text {th }}$ (grey) percentiles marked. 


$\begin{array}{lll}\text { df } & \boldsymbol{F} & P \\ \text { means square } & \end{array}$

\begin{tabular}{lccccc}
\hline Crop type & rice/wheat/barley & 2 & 0.0719 & 2.35 & 0.098 \\
$\begin{array}{l}\text { Shoot As level } \\
\text { Crop type } \\
\quad \text { X }\end{array}$ & $\log \mu \mathrm{g} \mathrm{g}^{-1}$ & 1 & 6.4732 & 212.01 & $<0.001$ \\
$\begin{array}{l}\text { Shoot As level } \\
\text { error }\end{array}$ & 2 & 0.3334 & 10.92 & $<0.001$ \\
\hline
\end{tabular}

Table S10. Analysis of variance of As grain level between rice, wheat and barley, with variable shoot As levels. Data was logged prior to analysis and 6 outliers were removed from the data set to normalise residuals. 


\begin{tabular}{ccccccc} 
& & $\begin{array}{c}\text { R sqrt } \\
\%\end{array}$ & y0 & a & p & n \\
\hline $\begin{array}{c}\text { soil } \\
\text { s. } \\
\text { shoot }\end{array}$ & rice & 30.8 & -0.35 & 1.07 & $<0.001$ & 73 \\
& wheat & 42.9 & -1.37 & 0.54 & $<0.001$ & 64 \\
& barley & 51.8 & -1.39 & 0.54 & $<0.001$ & 35 \\
\hline $\begin{array}{c}\text { soil } \\
\text { s. }\end{array}$ & rice & 17.1 & -1.04 & 0.39 & $<0.001$ & 72 \\
$\begin{array}{c}\text { grain } \\
\text { wheat }\end{array}$ & 41.5 & -2.03 & 0.52 & $<0.001$ & 64 \\
\hline $\begin{array}{c}\text { s. } \\
\text { grain }\end{array}$ & barley & 37.8 & -201 & 0.48 & $<0.001$ & 35 \\
\hline & wheat & 36.7 & -1 & 0.59 & $<0.001$ & 64 \\
\hline & barley & 28.7 & -0.98 & 0.56 & $<0.001$ & 35 \\
\hline
\end{tabular}

Table S11. Summary of regressions for Soil-Shoot-Grain As levels for rice, wheat and barley. $y 0=y$-axis intercept; $a=$ gradient; $p=$ significance from ANOVA; $\mathrm{n}=$ number of samples. 


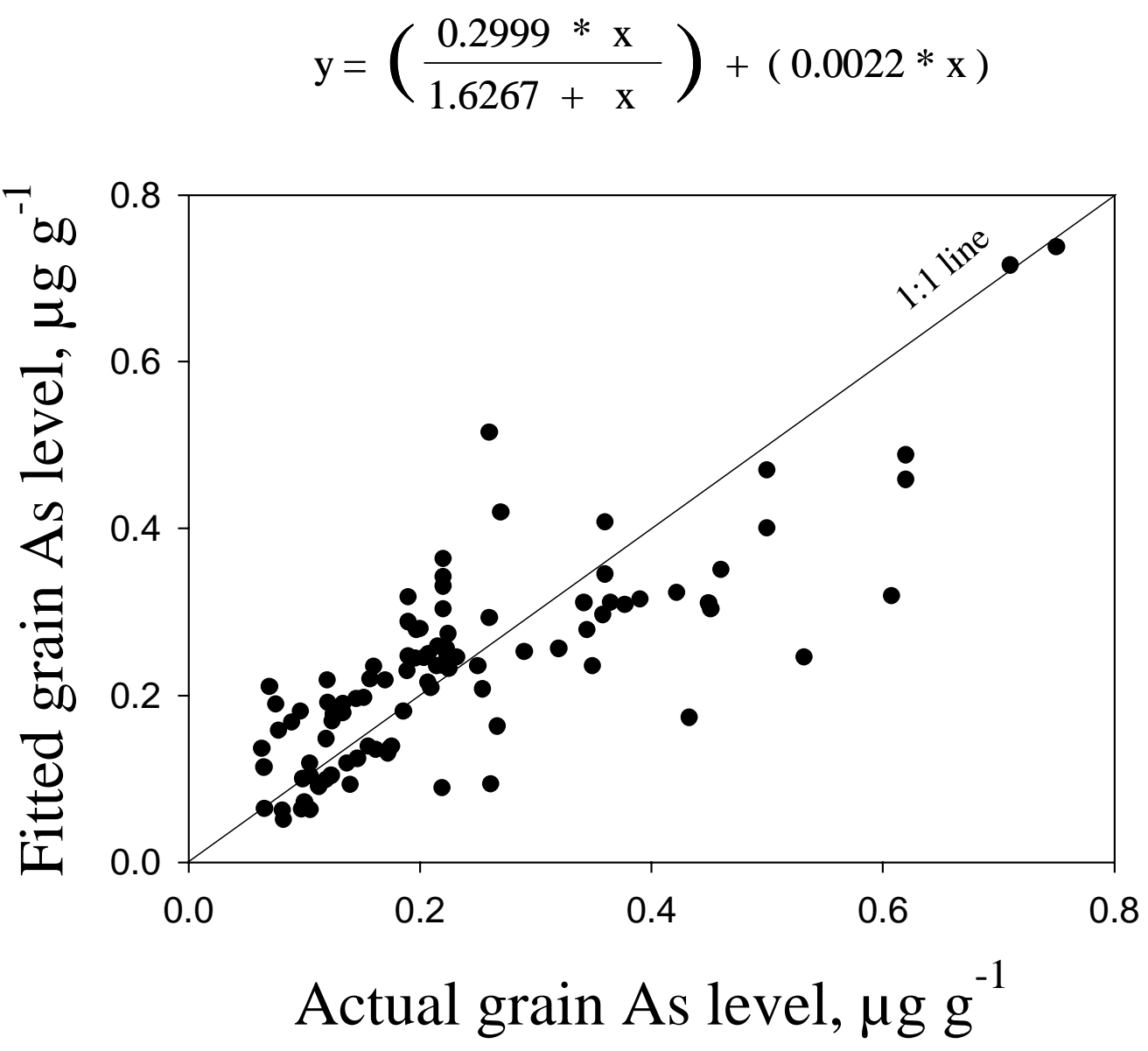

Fig S4. Predicting rice grain As level. A hyperbola regression model, derived from 96 samples, using shoot As level was employed to predict grain As levels. The $\mathrm{R}^{2}$ for the model was 0.63. $\mathrm{y}=$ grain As level, $\mu \mathrm{g} \mathrm{g}^{-1} \cdot \mathrm{x}=$ shoot As level, $\mu \mathrm{g} \mathrm{g}^{-1}$. 


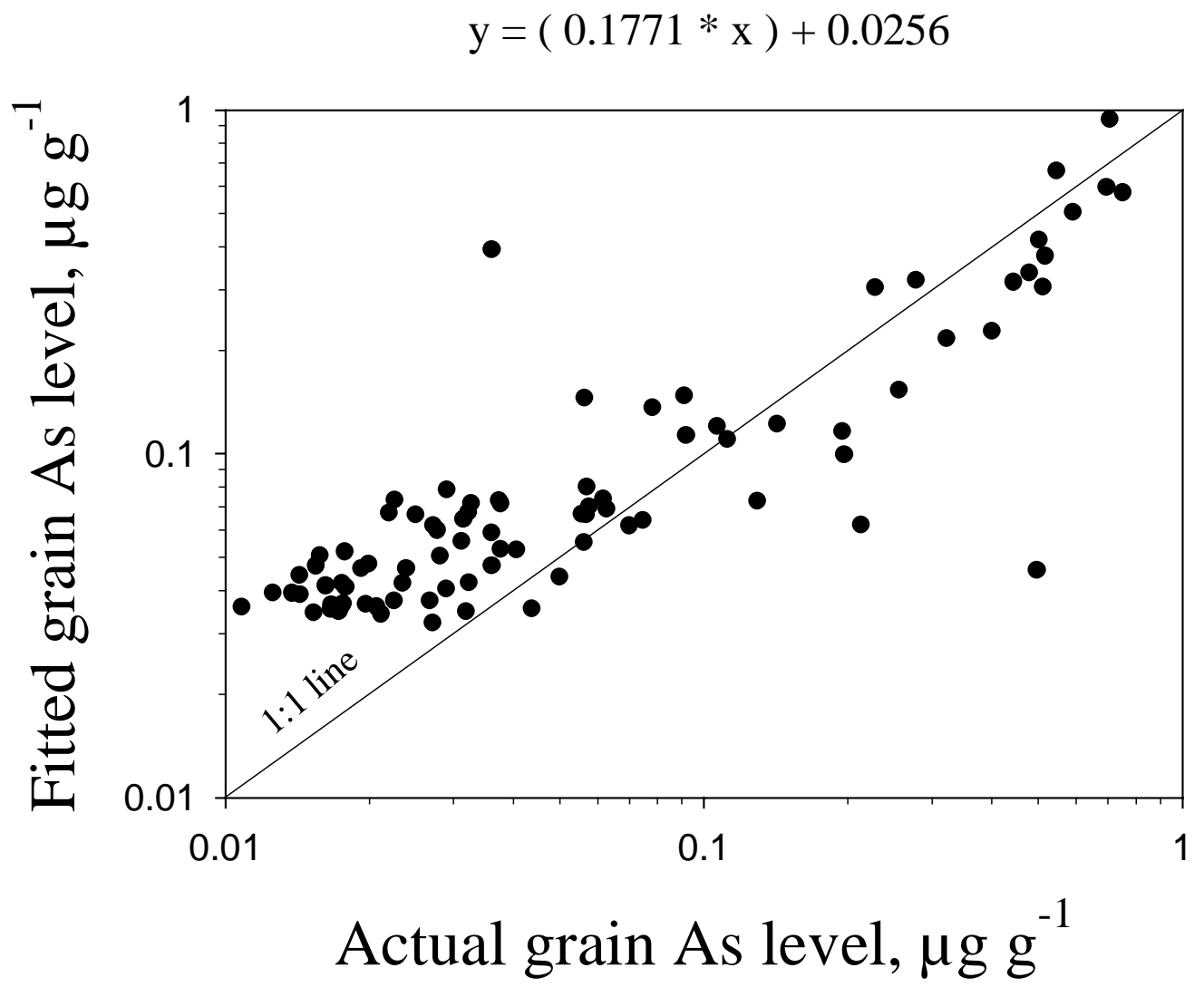

Fig S5. Predicting wheat grain As level. A linear model, derived from 87 samples, using shoot As level was employed to predict grain As levels. The $\mathrm{R}^{2}$ for the model was 0.76. $\mathrm{y}=$ grain As level, $\mu \mathrm{g} \mathrm{g}^{-1} . \mathrm{x}=$ shoot As level, $\mu \mathrm{g} \mathrm{g}^{-1}$. 


$\begin{array}{rc}\text { Soil pH (in water) } & 6 \\ \text { Total N }(\%) & 0.06 \\ \text { Organic carbon }(\%) & 0.75 \\ \text { CEC }(\mathrm{meq} / 100 \mathrm{G}) & 21.2 \\ \text { Exchangeable Ca }(\mathrm{meq} / 100 \mathrm{~g}) & 11.96 \\ \text { Exchangeable } \mathbf{M g}(\mathrm{meq} / 100 \mathrm{~g}) & 7.92 \\ \text { Exchangeable Na }(\mathrm{meq} / 100 \mathrm{~g}) & 0.77 \\ \text { Exchangeable K }(\mathrm{meq} / 100 \mathrm{~g}) & 1.04 \\ \text { Available P }(\mu \mathrm{g} / \mathrm{g}) & 4.6 \\ \text { Available Fe }(\mu \mathrm{g} / \mathrm{g}) & 26.1 \\ \text { Available } \mathbf{M n}(\mu \mathrm{g} / \mathrm{g}) & 33.5 \\ \text { Available Cu }(\mu \mathrm{g} / \mathrm{g}) & 0.87 \\ \text { Available } \mathbf{Z n}(\mu \mathrm{g} / \mathrm{g}) & 0.47 \\ \text { Total As }(\mu \mathrm{g} / \mathrm{g}) & 31\end{array}$

Table S12. Physico-chemical properties of soil used in the experiment, taken from Abedin et al. (3). 


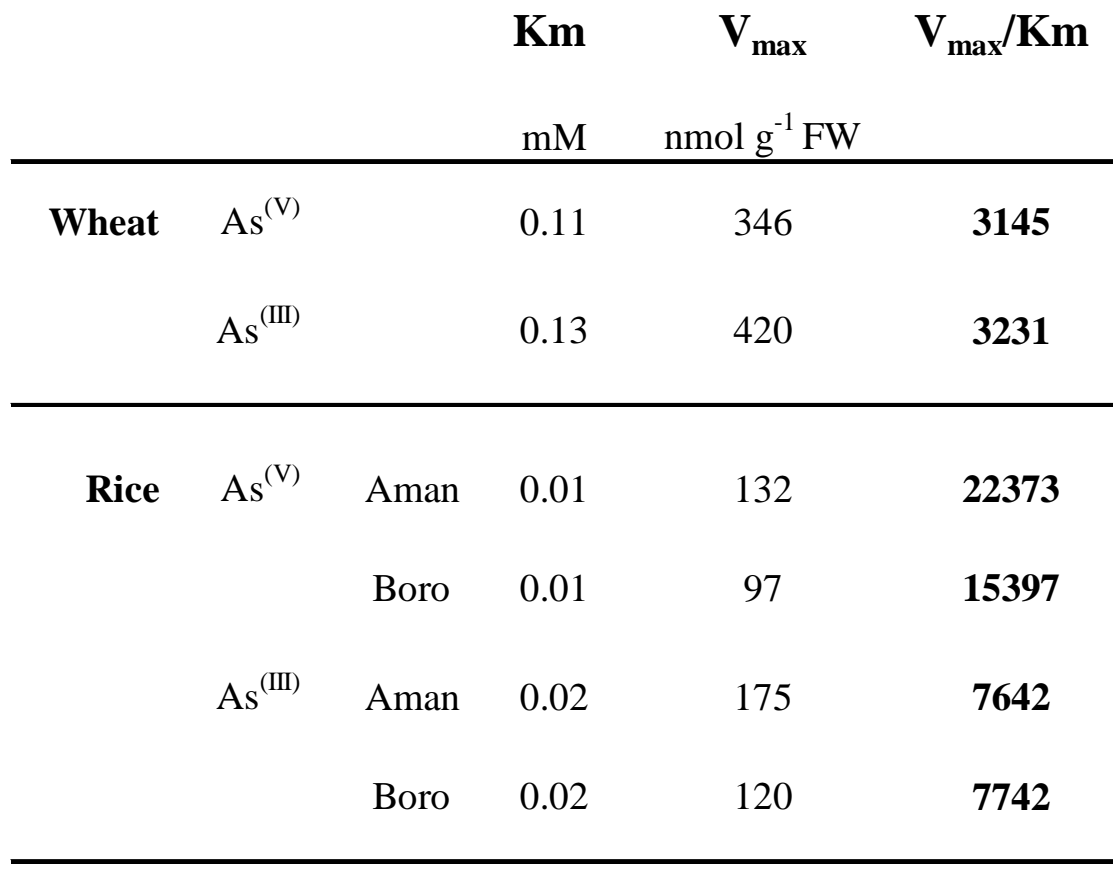

Table S13. Kinetic parameters for arsenate and arsenite influx in rice and wheat. Adapted from Abedin et al. (4) and Liu et al. (5). 


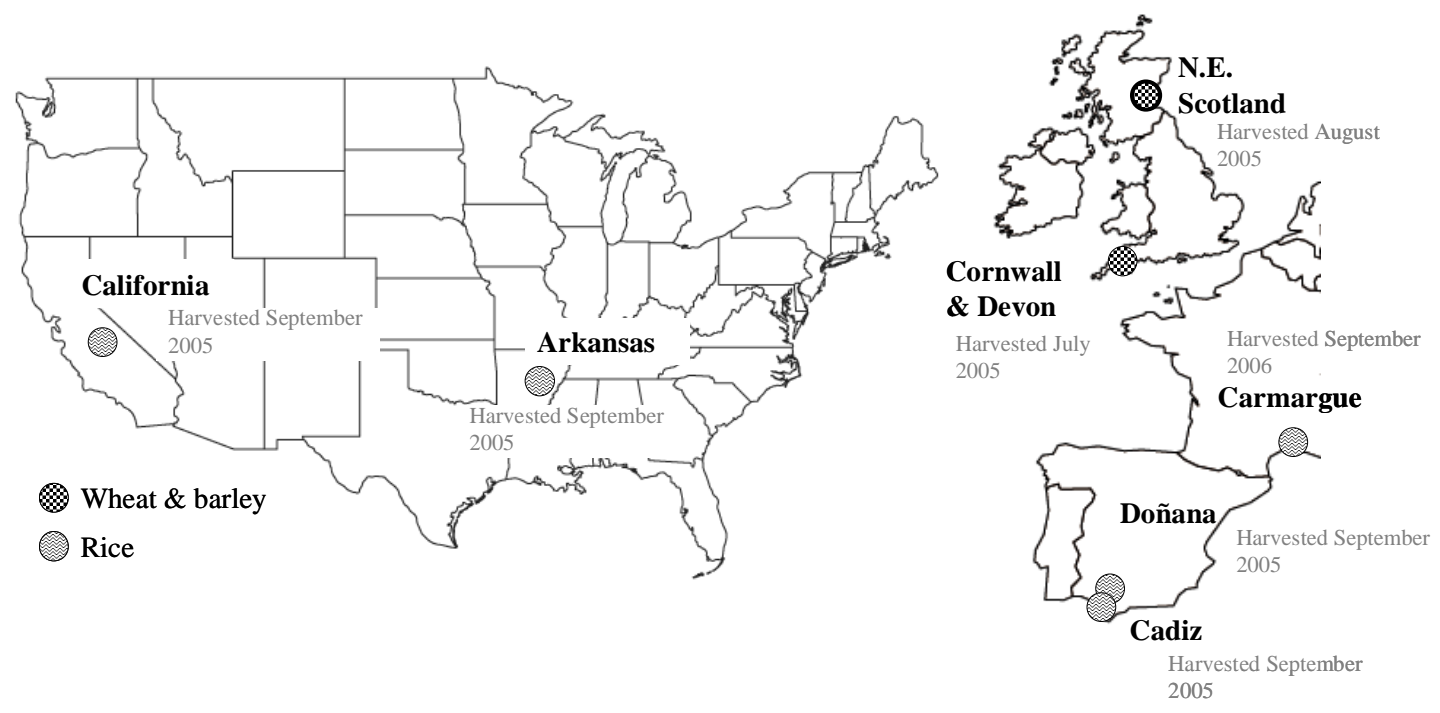

Figure. S6. Sample Map. Location and date of sampling. 


\section{References}

1) Singh, D.; Park, R.F.; McIntosh, R.A. Postulation of leaf (brown) rust resistance genes in 70 wheat cultivars grown in the United Kingdom.

Euphytica, 2001, 120, 205-218.

2) Jiang, W.Z.; Ireland, C.R. Characterization of manganese use efficiency in UK wheat cultivars grown in a solution cultures system and in the field. $J$. Agric.Sci. 2005, 143, 151-160.

3) Abedin, M.J.; Cotter-Howells, J.; Meharg, A.A. Arsenic uptake and accumulation in rice (Oryza sativa L.) irrigated with contaminated water. Plant Soil. 2003, 240, 311-319.

4) Abedin, M.J.; Feldmann, J.; Meharg, A.A. Uptake kinetics of arsenic species in rice plants. Plant Physiol. 2002, 128, 1120-1128.

5) Liu, X.; Zhang, S.; Shan, X.; Zhu, Y.-G. Toxicity of arsenate and arsenite on germination, seedling growth and amylolytic activity of wheat. Chemosphere. 2005. 61, 293-301. 